\title{
APPLICATION OF THE CEL APPROACH TO CONSIDER FSI FOR THE ASSESSMENT OF LEAK TIGHTNESS FOR ELASTOMERIC SEALS
}

\author{
Yevgen Gorash* \\ Mechanical \& Aerospace Engineering \\ University of Strathclyde \\ Glasgow, G1 1XJ \\ Scotland, United Kingdom \\ yevgen.gorashestrath.ac.uk
}

\author{
Alan Bickley \\ Weir Advanced Research Centre \\ Technology and Innovation Centre \\ Glasgow, G1 1RD \\ Scotland, United Kingdom \\ alan.bickley@mail.weir
}

\author{
Francisco Gozalo \\ Weir Rubber Engineering \\ Weir Minerals \\ Salt Lake City, UT 84119 \\ francisco.gozalo@mail.weir
}

\begin{abstract}
Swellable elastomeric seal is a type of specifically engineered packer that swell upon contact with wellbore fluids. Assessment of leakage tightness is a fundamental aspect in the design of swellable packers, since they should guarantee a reliable sealing under extreme pressures of the downhole fluids. Numerical capability of the leakage pressure prediction would facilitate improvement in the packer design methodology. Previous work was focused on investigation of the non-parametric optimisation capability seeking for an optimal external shape with a goal to maximise the grip of a packer with a borehole. The verification of an optimised design was done with a dynamic FE-simulation of packer's failure by extrusion under an excessive pressure. The downside of that verification analysis was that Abaqus/Explicit solver couldn't implement a realistic adaptive pressure application due to changing packer disposition and contact conditions. This simulation challenge is addressed in this paper by application of the Coupled Eulerian-Lagrangian (CEL) approach in Abaqus/Explicit, which provides the ability to simulate a class of problems where the fluid-structure interaction is important.
\end{abstract}

\section{NOMENCLATURE}

\section{Abbreviations}

BC Boundary Condition

CEL Coupled Eulerian-Lagrangian

FEA Finite Element Analysis

FSI Fluid-Structure Interaction

HPC High Performance Computing

PPL Pressure Penetration Load
Variables, Constants

$\mu_{i}, \alpha_{i}(i=1 . .3) \quad$ parameters for Ogden hyperelastic model

$\mu, \lambda_{m}, \alpha, \beta \quad$ parameters for van der Waals hyperel. model

$D$

$K_{0}$ and $\mu_{0}$

$\rho$ and $v$

$s$ and $c_{0}$

$\eta$ and $F_{n}$

$F_{\tau}$ and $\tau_{c}$ compressibility parameter

initial bulk modulus and initial shear modulus material density and Poisson's ratio dynamic viscocity and speed of sound coefficient of friction and normal force critical share force and critical shear stress

\section{INTRODUCTION}

Swellable elastomeric seal is a type of specifically engineered packer that swell upon contact with wellbore fluids. Such packers have been widely employed in various oil-\&-gas applications including slimming of well design, zonal isolation, water shut-off, and multi-stage fracturing. Assessment of leakage tightness is a fundamental aspect in the design of swellable packers, since they should guarantee a reliable sealing under extreme pressures of the downhole fluids up to $10 \mathrm{ksi}(69 \mathrm{MPa})$. Downhole conditions are difficult to be reproduced using physical testing environment, but feasible to be simulated [1] in virtual environment using FE-codes. Numerical capability of the leakage pressure prediction under different downhole conditions (type of downhole fluid, pressure build-up rate, diameter of the borehole, etc.) would facilitate improvement in the packer design methodology and would allow efficient optimisation of a packer design. Previous work $[2,3]$ was focused on investigation of the nonparametric optimisation capability seeking for an optimal external shape with a goal to maximise the grip of a packer with a borehole by maximising the contact pressure between them. For

*Address all correspondence to this author. 


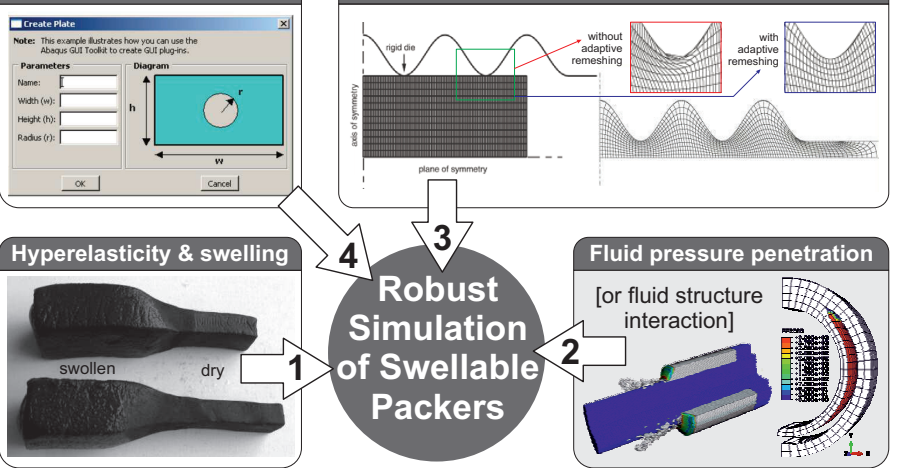

FIG. 1 COMPONENTS OF ROBUST FE-SIMULATION OF SWELLABLE PACKERS FOR FAILURE AND LEAKAGE

this purpose, Tosca/Structure optimisation suite was used within the Abaqus/CAE environment for maximum computational performance. The verification of an optimised design was done through the dynamic FE-simulation of packer's failure by extrusion under an excessive pressure. The downside of that verification analysis was that Abaqus/Explicit solver couldn't implement a realistic adaptive pressure application due to changing packer disposition and contact conditions. This simulation challenge is addressed in this work by application of the Coupled Eulerian-Lagrangian (CEL) approach in Abaqus/Explicit, which provides engineers with the ability to simulate a class of problems where the fluid-structure interaction (FSI) is important, like seals. This capability does not rely on the coupling of multiple software products, but instead solves the FSI simultaneously within single Abaqus environment. The most relevant example of the CEL application to investigation of leakage tightness is the study [4], where the CEL approach predicts not only the pressure at which the seal blows off, but also how the fluid behaves when leakage starts. Apart from the technology demonstration [4], there is a very limited availability of literature sources focused on FSI modelling, that combines extremely large deformations of hyperelastic structures with CEL to address changing contact conditions between fluid and structure.

The idea similar to the one implemented in [4] lies beneath the given numerical study - to investigate a feasibility of FSI simulation with CEL in application to failure analysis of swellable packers. The feasibility assessment would ideally include the computational costs and robustness level of this type of analysis considering the specific conditions including incompressible nature of the material, high pressure applied as a loading and extremely large deformations as a result of excessive pressure application.

\section{BACKGROUND}

In general, the objective of this research project is to develop a design tool integrated into Abaqus/CAE environment to implement the parametric numerical studies using advanced FEsimulation to provide an improved design of packers for vari- ous downhole conditions. However, the implementation of the packer's swelling and failure simulations is associated with a number of technical/numerical challenges specific to this particular class of multiphysics problems, which are illustrated in Fig. 1 and listed below:

1. Material model. The key component is an advanced material model comprising both hyperelasticity and moisture swelling. It has to consider two-way interaction between mechanical response and swelling capacity. Implementation of such a material model requires using COMSOL Multiphysics [5] or programming of a Fortran subroutine for the user defined material using the Flory \& Rehner (1943) theoretical background [6].

2. Fluid-structure interaction. The moisture swelling process is not uniform and starts on the surfaces which are subject to fluid. Adsorption, which governs the progress of swelling can occur only at free surfaces. Therefore, the fluid pressure penetration needs to be incorporated into the simulation [7] and directly linked to swelling. Distributed pressure penetration load allows for the simulation of fluid penetrating into the surface between two contacting bodies, penetration of fluid from multiple locations on the surface, and application of the fluid pressure normal to the surfaces. It automatically adjusts the application of a fluid pressure depending on changes of contact conditions.

3. Large deformation convergence. Non-uniform swelling is associated with a localised increase of material volume. It may cause a significant distortion of FE mesh and arouse FEA convergence problems. To overcome this, there are a few options available in the setup of the FEmodel [8] including a mesh-to-mesh solution mapping (Abaqus/Standard), adaptive remeshing (Abaqus/Explicit) and element distortion control. Convergence issue is crucial to the successful solution of elastomeric structures FEsimulation, because in most cases the FE-analysis fails because of excessive distortion or collapse of elements.

4. Parametric analysis automation. Parametric study assumes considering a large number of different geometric configurations, looking at material properties variation and different downhole conditions. Basically this means a search for an optimal geometry through a sensitivity study, which would result in specific design recommendations for the geometry of a packer. Therefore, it would be reasonable to automate the analysis procedure through an Abaqus plug-in [9] with a convenient graphical user interface (GUI), which provides access to the parameters of geometry, material properties and service conditions.

\section{VALIDATION OF SHAPE OPTIMISATION}

In previous works [2,3] the feasibility of non-parametric optimisation [10] in application to swellable packers was investigated following the successful outcomes of [11] that revealed a great potential of the topology and shape optimization under contact conditions. For that purpose, Simulia Tosca Structure was 

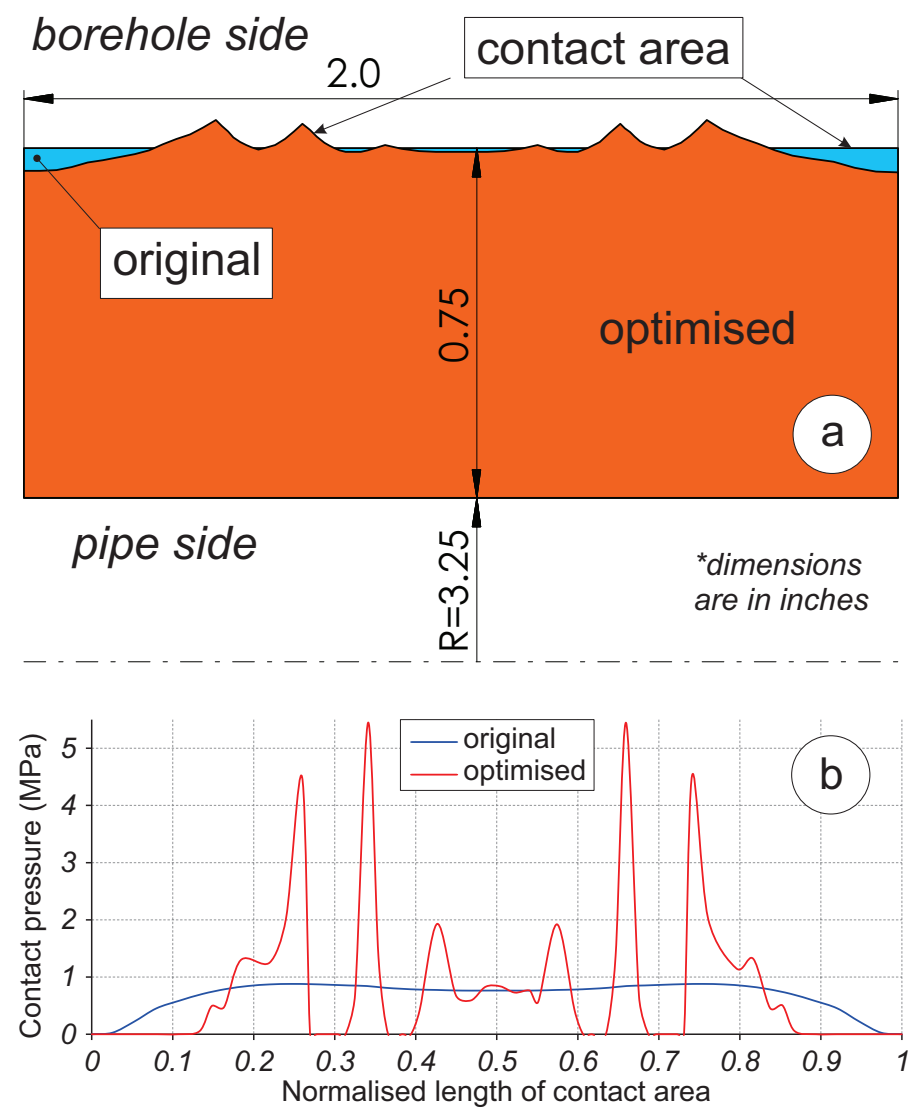

FIG. 2 SHAPE OPTIMISATION OF THE PACKER PROFILE: A) CHANGE OF PROFILE GEOMETRY AND B) CORRESPONDING CHANGE OF CONTACT PRESSURE

used - a software system for non-parametric structural optimisation with interfaces to the most of industry standard FE-solvers. Using optimisation techniques, contact pressure in contact zones could be either minimised [12] or maximised as needed in this research. Therefore, the shape optimisation was used [2,3] to improve the grip of a packer with the surface of a borehole. The normal shape optimization stimulated the surface growth in contact zones, which resulted in a higher contact pressure and shrinkage in a lower. For a trial shape optimisation study, the trimmed version of a packer geometry [1] was used as benchmark problem with $L$ reduced from 16 " to 2". The optimisation analysis resulted in a rippled external surface of a packer as shown in Fig. 2a with comparison to the original rectangular profile. The distribution of contact pressure became very non-uniform as shown in Fig. $2 b$ with four maximums, which are about 5 times higher than the original smooth contact pressure.

An important part of optimisation analysis is a validation of the obtained design, which in this study is expressed in terms of comparative sealing capability. The basic qualitative validation analysis was performed using the general static simulation procedure with implicit solver in Abaqus/Standard [2]. For a more comprehensive and quantitative validation of the packer design, the simulation capabilities of Abaqus/Standard solver were found insufficient. The advantage of Abaqus/Standard implicit solver was a fast solution and the availability of PPL interaction [7]. This functionality replaces the computationally expensive fluid-structure interaction, when the structural analysis has a priority. On other hand, the disadvantage of implicit solver is that the automatic adaptive remeshing is not available as a standard functionality, so the extrusion problems with extreme deformation can't be effectively solved using this product. Therefore, the subsequent work [3] was implemented with the dynamic solver in Abaqus/Explicit, which is recognised as a more robust solver when it comes to very non-linear problems and extremely large deformations.

Abaqus/Explicit was computationally more expensive compared to Abaqus/Standard, but this obstacle was overcome by running simulations on HPC facility. This solver significantly expands the progressive failure analysis capabilities, and actually eliminates any limitations related to non-linearities, large deformations and transient / dynamic effects. The best prove of its efficiency is a solution of a so-called press-fit problem [13], when a cylindrical rubber block compressed from the tube of bigger diameter into the tube with a smaller diameter. In previous work [14] an attempt to develop a robust approach to simulation has failed. A simple and stable solution for such a benchmark problem using standard implicit solvers in Ansys and Abaqus couldn't be obtained. It should be noted that the successful simulation of press-fit problem [13] became possible only after the modification of a friction model used in analysis from the linear Coulomb to the bi-linear Coulomb-Orowan law [15] expressed in terms of friction force as

$$
F_{f}=\min \left(\eta\left|F_{n}\right|, F_{\tau}\right)
$$

where $\eta$ is a coefficient of friction, $F_{n}$ is a normal force, and $F_{\tau}$ is a critical share force, which corresponds to a critical shear stress $\tau_{c}$ in the FEA setup. The Coulomb term $\eta\left|F_{n}\right|$ is linear and describes the partial slip. When the critical value of $\tau_{c}$ is reached, the total slip occurs, which plays a key role in simulation convergence, because it prevents the rubber material from sticking to the relatively rigid walls.

So the work [3] was focused on the development of a practical approach to simulations of packers with Abaqus/Explicit, since the setup of analyses in Standard and Explicit solvers is quite different. The biggest advantages attributed to Explicit solver are automatic adaptive remeshing (in application to large plastic deformations) or distortion control of elements (in application to large hyperelastic deformations) and stable solution of contact problems with large relative displacements. Considering a superior robustness of Abaqus/Explicit, it is a minor drawback that PPL functionality is unavailable for dynamic analysis. The robustness of extrusion failure simulations for swell packers were demonstrated in [3] with advanced validation analysis of the benchmark problem.

Since PPL is unavailable, the pressure was applied to the bottom surface and ramped in the course of simulation for both benchmark packers - original and optimised. The stable and ro- 

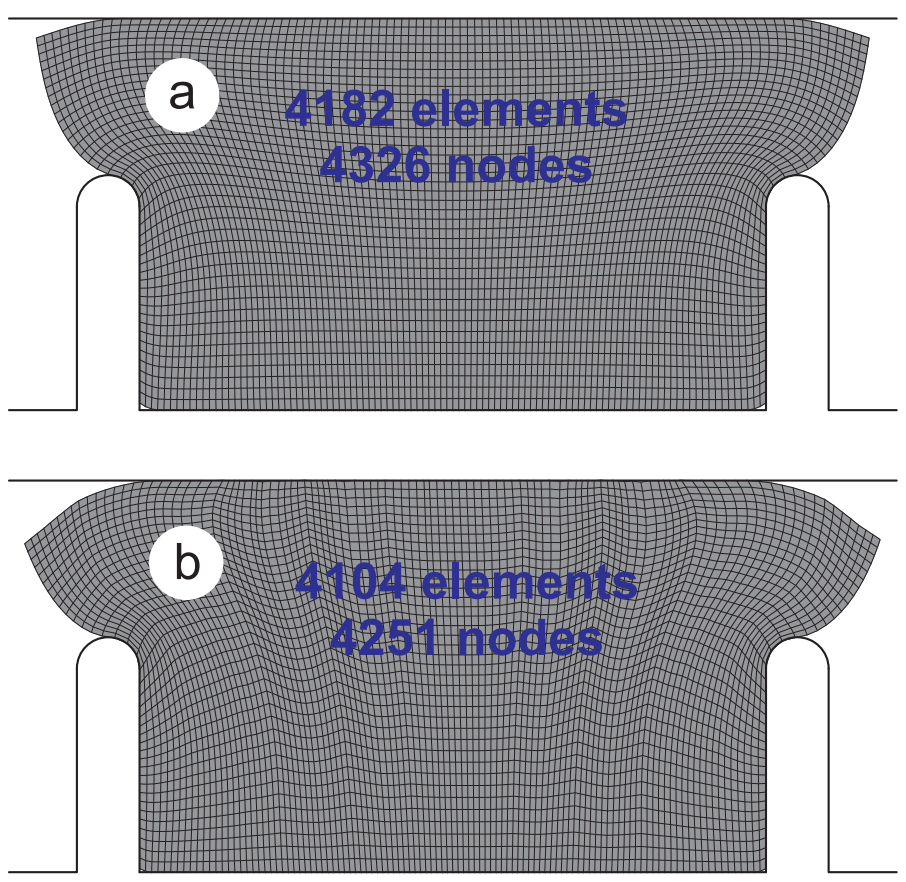

FIG. 3 FE-MESHES OF (A) ORIGINAL AND (B) OPTIMISED BENCHMARK PACKER GEOMETRIES

bust convergence has been achieved with the CAX3 element type - a 3-node linear axisymmetric triangle with the activated distortion control having length ratio 0.5 . This means that the FEmodel topology is adjusted when an element under uniaxial compression undergoes $50 \%$ of nominal strain. This FE-mesh adjustment technique [8] together with the bi-linear Coulomb-Orowan friction law provides a guaranteed convergence of a dynamic solution in Abaqus/Explicit. The absence of hourglass issues is provided automatically by the triangular shape of the elements. In should be also noted that in order to accelerate the analysis and facilitate the convergence the default Abaqus/Explicit compressibility ratio (initial bulk modulus to initial shear modulus) $K_{0} / \mu_{0}=20$ has been used [3], corresponding to Poisson's ratio $v$ of 0.475 . Since typical unfilled elastomers have $K_{0} / \mu_{0}$ ratios in the range of 1,000 to $10,000(v=0.4995$ to $v=0.49995)$ and filled elastomers have $K_{0} / \mu_{0}$ ratios in the range of 50 to 200 ( $v$ $=0.490$ to $v=0.497$ ), this default provides much more compressibility than is available in most elastomers [16]. The forced incompressibility will become more feasible in the future version of ABAQUS (2018) with introduction of the hybrid formulation for elements used in Abaqus/Explicit solver.

Comparison of simulation results showed [3] that the optimised packer can bear about $10 \%$ of more pressure compared to the original packer with a smooth surface providing and additional validation of optimisation results. The validation simulation of a full-size real packer [1] demonstrated a complete extrusion of the packer [3]. It also showed that extrusion was not gradual, it was rather abrupt with a distinctive critical pressure when sticking to protective rings can't stop progressive slipping, caused by friction and material compressibility. With the recent findings related to convergence facilitation techniques,
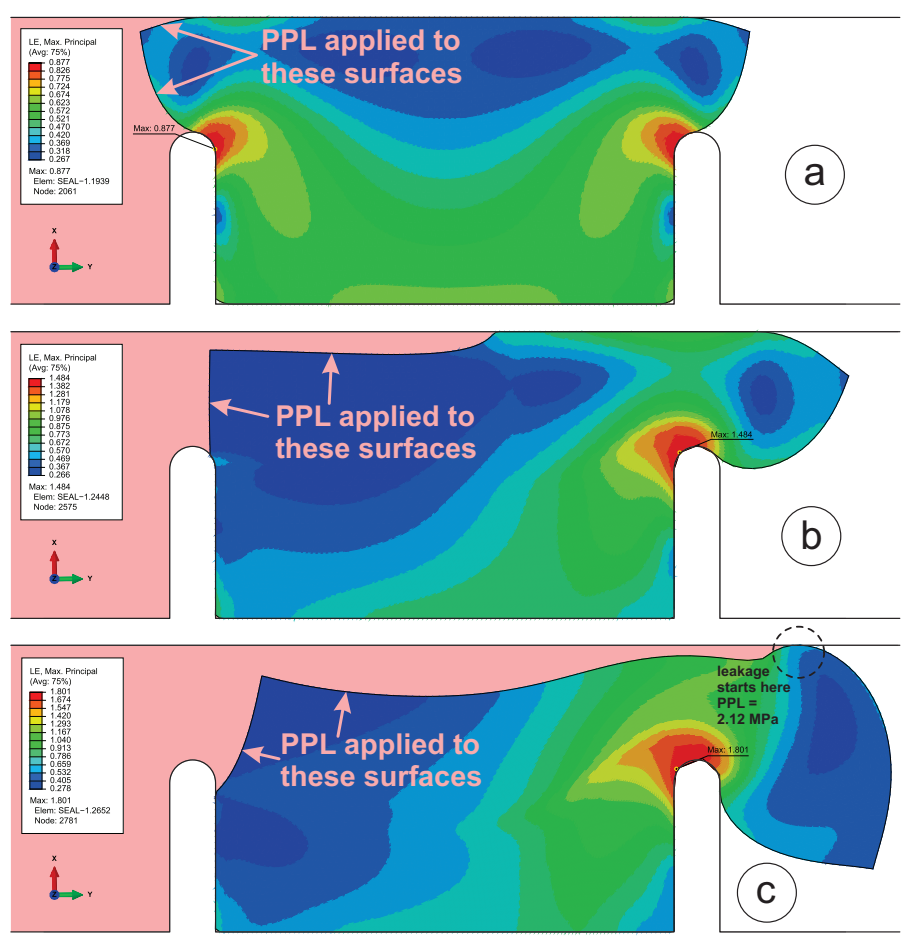

FIG. 4 VALIDATION OF BENCHMARK PACKER FAILURE USING ORIGINAL GEOMETRY WITH ABAQUS/STANDARD \& MESH DISTORTION CONTROL

it was decided to revisit the static implicit simulations with Abaqus/Standard [2] in a view of limited analysis functionality in terms of realistic incompressibility and load application. Figure 3 shows the FE-meshes of (a) original and (b) optimised benchmark packer geometries consisting of the CAX4R element type, 4-node bilinear axisymmetric quadrilateral with reduced integration and distortion control having length ratio 0.5 .

The material parameters for van der Waals hyperelastic model were taken from [17] with following values: $\mu=0.385$, $\lambda_{m}=10.35 ; \alpha=0.279, \beta=0.95$ and $D=0.001$. They are based on Treloar's experimental set of stress-strain data for vulcanised rubber [18]. Since not a triangular-shaped element type was used in analysis with incompressible material, it required an additional hourglass control to stabilise its behaviour at very large strains. The stiffness hourglassing control has been used with the stiffness coefficient of 50, which provided a robust convergence for the benchmark packers simulations with results shown in Figs 4 and 5. In definition of general static step, the automatic adaptive solution stabilisation with specified dissipated energy fraction (0.0002) and maximum ratio of stabilisation to strain energy (0.05) were used. In this case, the combination of Coulomb-Orowan friction law, element distortion control, stiffness hourglassing control and automatic adaptive solution stabilisation helped to achieve a stable simulation of elastomeric component in axisymmetric formulation.

The failure modes of original and optimised packers are significantly different as can be seen from Figs $4 \mathrm{c}$ and $5 \mathrm{e}$. The burst pressure in case of optimised packer is also 30\% higher than for 

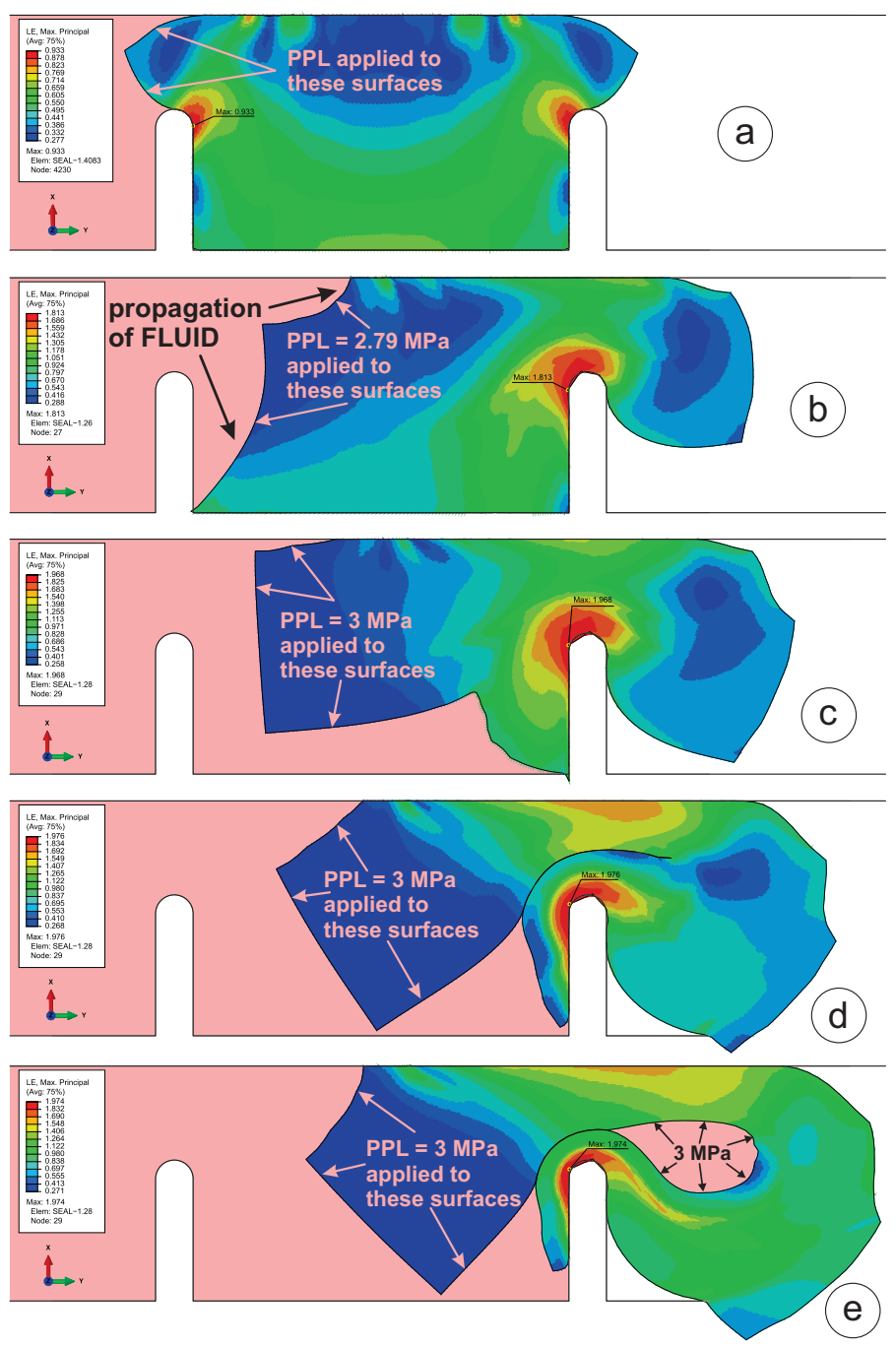

FIG. 5 VALIDATION OF BENCHMARK PACKER FAILURE USING OPTIMISED GEOMETRY WITH ABAQUS/STANDARD \& MESH DISTORTION CONTROL

original one (2.12 MPa vs $3 \mathrm{MPa}$ ). The leakage for the original packer occurs in a trivial and predicted way with a fluid pressure burst through the opened contact between packer and borehole as illustrated step-by-step in Fig. 4. When pressure builds up, the fluid propagates only in one direction parallel to axis Y. When reaching critical pressure, the packer gets partly extruded through the gap between the protective ring and borehole.

Failure mode of the optimised packer is significantly different and occurs in non-trivial way as illustrated step-by-step in Fig. 5. When pressure builds up, the fluid propagates in two directions as specified in Fig. $5 b-$ above the packer (through the opened contact between packer and borehole) and underneath the packer (the opened contact between packer and pipe). Contact opening above the packer lost its priority for fluid penetration because of the stronger grip between packer and borehole induced by the rippled packer surface. So it is easier for fluid to propagate in contact opening between packer and pipe, because of less contact pressure and less friction (see Fig. 5c). This scenario results in complete separation of packer from pipe and almost it's complete extrusion through the gap between the protective ring and borehole (see Fig. 5d). The extrusion is progressive and happens quickly almost without increase of pressure, when packer collapses approximately in its middle location and folds. It should be noted that even following the extrusion of the packer, the start of leakage is not explicit. The fluid pressure penetration results in the formation of cavity filled with fluid in the location of packer folding (see Fig. 5e). The cavity just goes on filling with fluid and growing without indication of pressure burst into outer space. The simulated scenario may seem unrealistic, because the packer should fail and rupture before filling with fluid. But this effect can be implemented only with inclusion of progressive material damage.

This numerical simulation finding indicates an interesting structural behaviour effect, which is worth of further investigation, because it may result in a potential design improvement. Regarding the validation analysis of the full-size packer [1], unfortunately it is still not feasible even with recently discovered convergence improvement techniques. Moreover, current and previous studies [2] showed that leakage is not static, it is a rather dynamic process accompanied by the formation of fluid cavities, their expansion and coalescence. Therefore, the dynamic analysis procedure supposed to be more adequate for realistic structural behaviour simulations of full-size packers.

\section{FLUID-STRUCTURE INTERACTION}

Since in previously conducted dynamic validation simulations with Abaqus/Explicit [3] interaction with fluid was not considered, they are lacking a realism, because the packer failure mode with a leakage through the contact surface can't be modelled. This simulation challenge can be addressed by an application of the Coupled Eulerian-Lagrangian (CEL) approach in Abaqus/Explicit, which provides engineers with the ability to simulate a class of problems where the interaction between structures and fluids is important. This capability does not rely on the coupling of multiple software products, but instead solves the fluid-structure interaction (FSI) simultaneously within the single Abaqus environment [19]. The potential of CEL approach for packers' leakage simulation is investigated below. The highest level of realism in simulation of leakage process is expected from engaging CEL in ABAQUS/Explicit. In order to develop a practical approach to the solution of this class of FSI problems and to understand corresponding capabilities and challenges, a leakage benchmark problem has been developed with the geometry shown in Fig. 6 (all dimension in $\mathrm{m}$ ). The assembly includes the following components:

1. computational fluid domain;

2. initial fluid volume;

3. rigid stationary walls (top, bottom, back);

4. rigid moving plunger;

5. deformable rubber seal constrained to the bottom wall.

The idea of this benchmark is to build up a fluid pressure by moving the plunger towards the seal in order to induce a progres- 


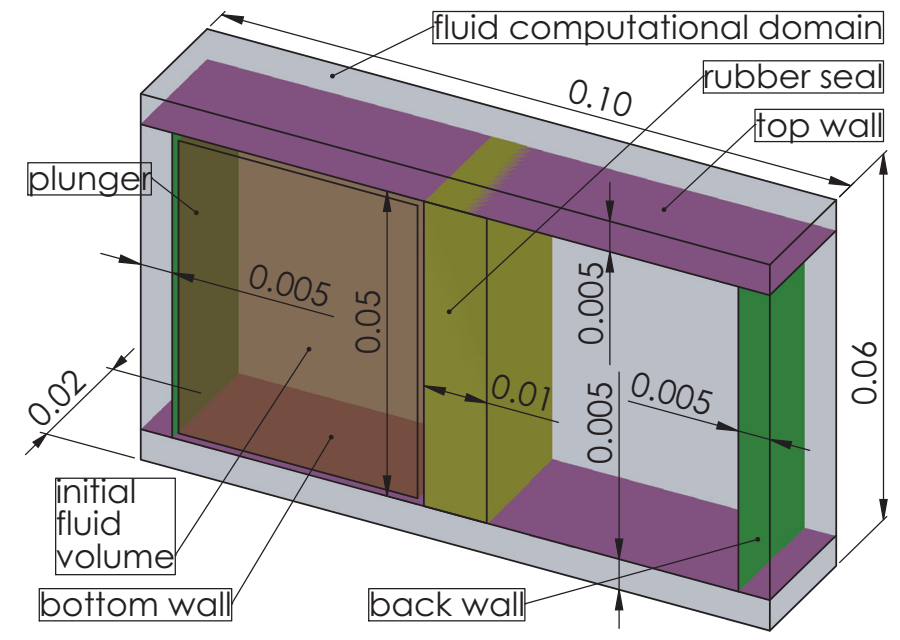

FIG. 6 THE DIMENSIONS (IN METERS) OF CEL BENCHMARK PROBLEM FOR SIMULATION OF LEAKAGE THROUGH A RUBBER SEAL AND IDENTIFICATION OF THE CORRESPONDING BURST PRESSURE

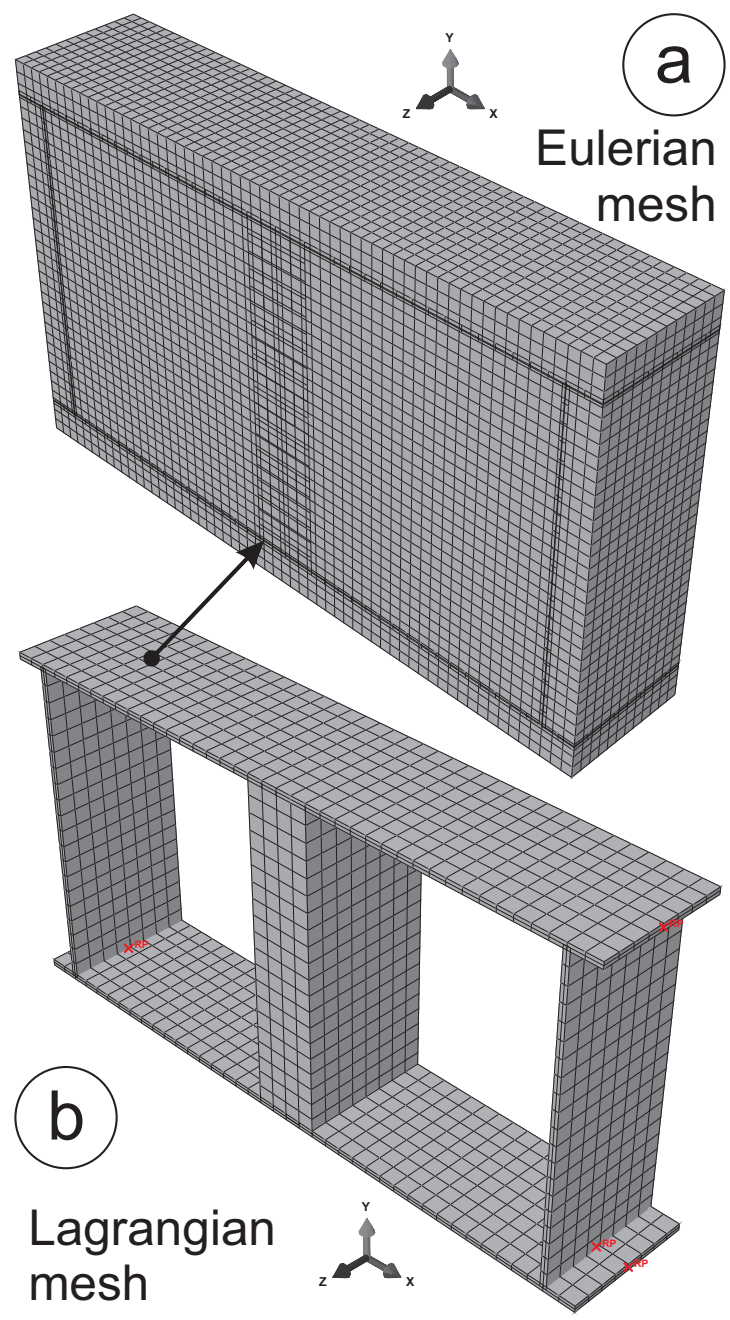

FIG. 7 FE-MESH OF COMPUTATIONAL FLUID DOMAIN AND SOLID PARTS ENCAPSULATED IN IT

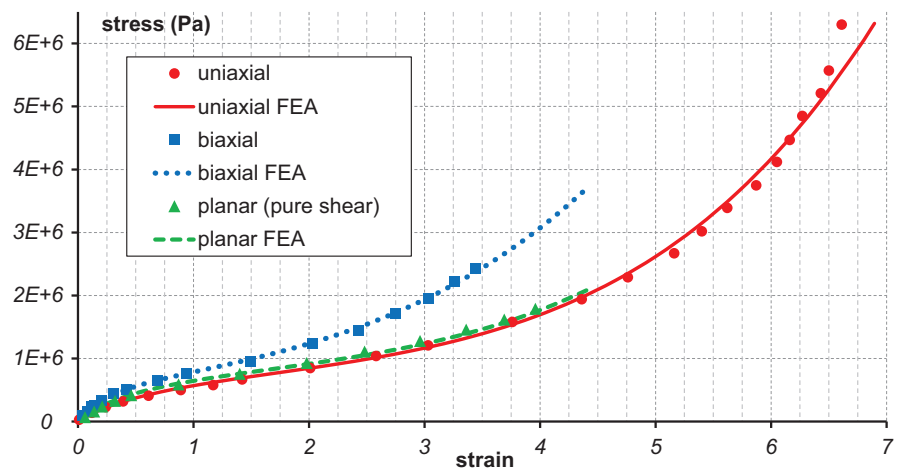

FIG. 8 RUBBER STRESS-STRAINS CURVES FITTED WITH THE 3RD ORDER OGDEN MODEL

sive deformation of rubber and subsequent leakage through the gap between deformed seal and top wall. All the fluid should be gradually displaced from the left cavity into the right void during the course of simulation. The fluid pressure is monitored during this process, so that the value of a burst pressure is identified by associating it to the moment in time, when the leakage occurs for the first time. It should be noted that for a simplicity the right cavity is considered to be a void in this study. For more realism, a presence of air can be considered in future simulations using separate initial Eulerian volumes and properties definition for a fluid and for a gas. The FE-model shown in Fig. 7 comprises the following type of elements:

- 24780 fluid Eulerian FEs (type EC3D8R) / 28080 nodes (water),

- 640 solid Lagrangian FEs (type C3D8R) / 945 nodes (rubber seal),

- 960 rigid shell FEs (type S4R) / 1116 nodes (rigid walls).

Since the number of Eulerian FEs exceeds almost 40 times the number of Lagrangian FEs, it is Eulerian part of the model, that is the most computationally expensive. The fluid element type, EC3D8R - 8-node linear Eulerian brick with reduced integration and hourglass control, is the only available type of fluid FE for CEL. The seal is meshed with C3D8R - 8-node linear brick with reduced integration, distortion control (length ratio 0.1 ) and enhanced hourglass control, which is quite sufficient to model an incompressible hyperelastic material undergoing moderate deformations. Since the hyperelastic materials parameters are required to be in SI units to avoid compatibility issues with the fluid material model, a new parameters identification has been implemented using internal Abaqus curve fitting tool [20] using Treloar's experimental set [18] with stress in Pa. In terms of strain energy potential, the 3rd order of Ogden form has been used resulting in the fit shown in Fig. 8 and the following set of parameters: $\mu_{1}=371784.2[\mathrm{~Pa}], \alpha_{1}=1.45175, \mu_{2}=1308.63$ $[\mathrm{Pa}], \alpha_{2}=5.4886, \mu_{3}=15445.055[\mathrm{~Pa}], \alpha_{3}=-1.87468, D=$ $5.1477 \cdot 10^{-10}$. The elastic strain observed in the seal is not rally high - just $\sim 1 \%$ when pressure starts to build up (see Fig. 9a), then it goes up to $6 \%$ when the leakage starts (see Fig. 10a), and it further increases up to $13.5 \%$ (see Fig. 9b) when the fluid flow 

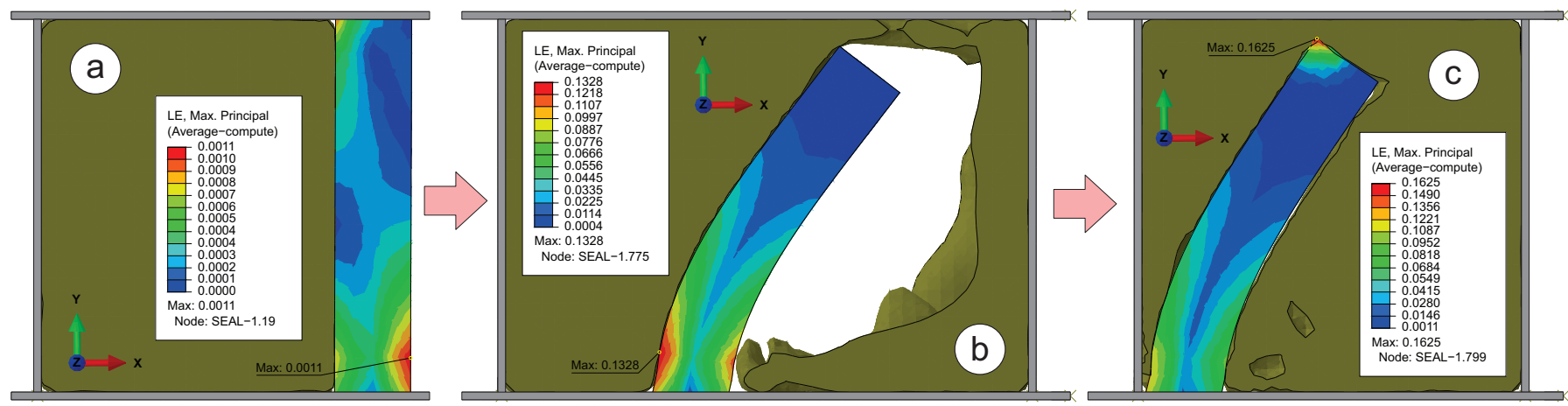

FIG. 9. TRUE STRAIN IN SEAL DURING: (A) PRESSURE BUILD-UP, (B) PROGRESSION OF FLOW AND (C) FLOW CUT-OFF
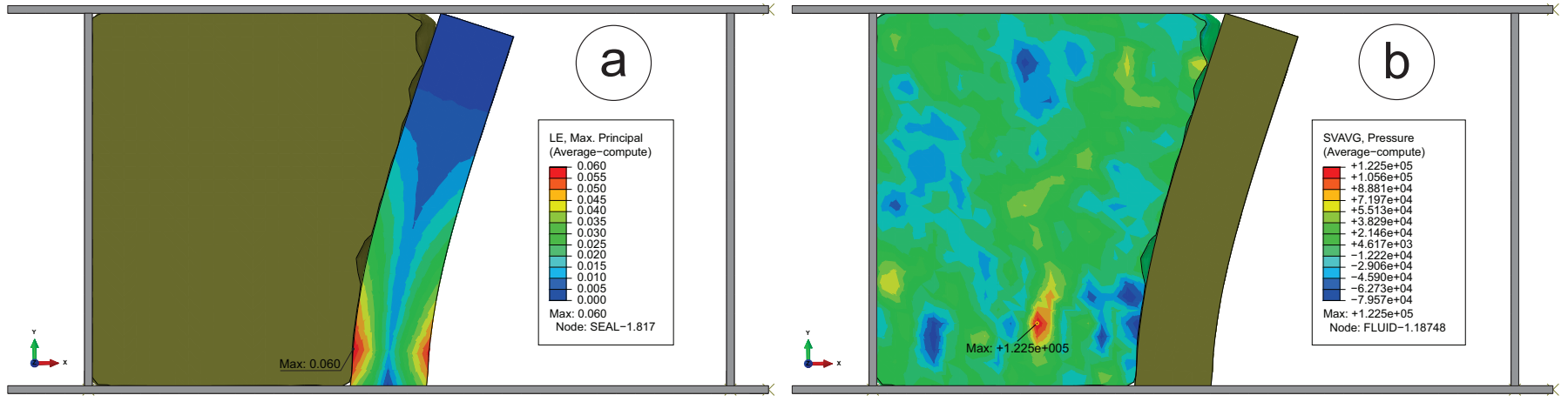

FIG. 10 THE MOMENT OF TIME CORRESPONDING TO THE START OF LEAKAGE CORRESPONDING TO (A) MAXIMUM PRINCIPAL TRUE STRAIN IN SEAL AND (B) PRESSURE DISTRIBUTION IN WATER

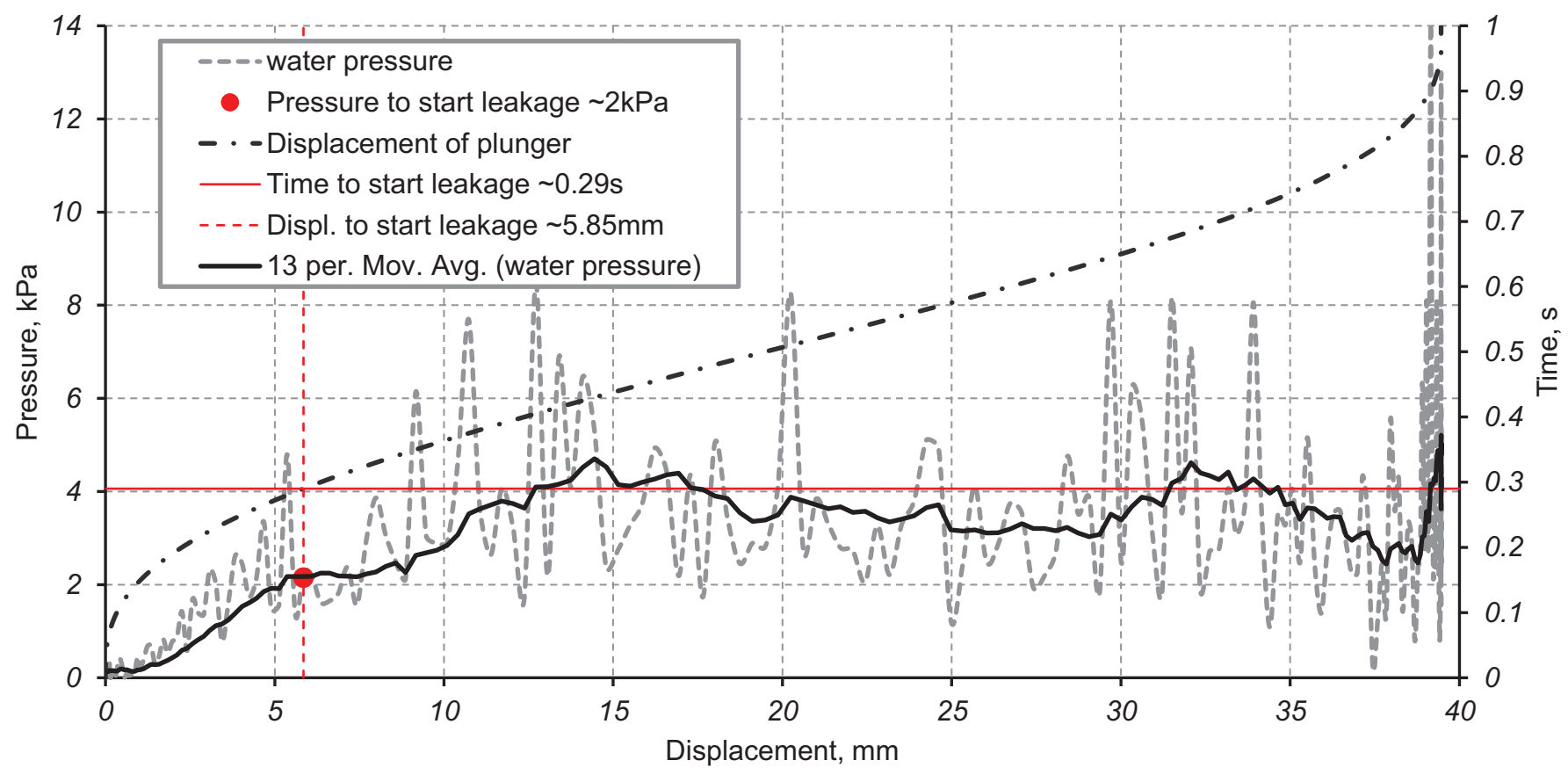

FIG. 11. AVERAGED FLUID PRESSURE (KPA) VS DISPLACEMENT OF PLUNGER (MM) VS TIME (S) 


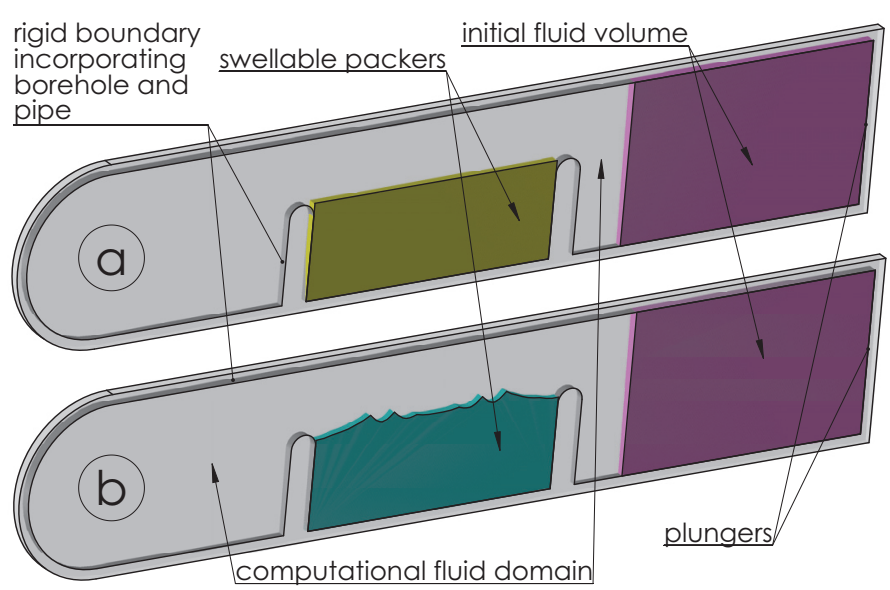

FIG. 12 GEOMETRIES OF THE PACKER BENCHMARK PROBLEMS: A) ORIGINAL AND B) OPTIMISED

becomes steady-state, and finally reaches $16 \%$ (see Fig. 9c) when the relocation of the fluid is finished.

The fluid material properties used in CEL simulation are based on the linear $U_{s}-U_{p}$ Hugoniot form of Mie-Grüneisen equation of state model [21], which is used to model compressible viscous and inviscid laminar flow governed by the NavierStokes equation of motion. The definition of a material using this material model requires the speed of sound in a medium $c_{0}$ and the dynamic viscosity $s$, which are in this case taken as a general case corresponding to water at room temperature $-c_{0}=1483$ $[\mathrm{m} / \mathrm{s}]$ and $s=0.001[\mathrm{~Pa} \cdot \mathrm{s}]$ with the density $\rho=1000[\mathrm{~kg} / \mathrm{m} 3]$ for dynamic FEA.

\section{DISCUSSION}

The obtained water pressure distribution is quite noisy spatially as seen from Fig. 10b with random peaks and valleys (maximums and minimums), which can be traced during the simulation. Since the volumetric variation of pressure is very significant, it order to get a better understanding of its change over the time, a solution would be to average the pressure across the entire domain at each analysis substep. However, there are $\sim 25000$ elements in the Eulerian domain (see Fig. 7a), and each element has a value of averaged pressure for each of the 200 available output substeps. Since ABAQUS is not optimised for doing such big postprocessing jobs, as a directly available working solution, the pressure data for all Eulerian elements in the analysis is exported to a text file and further processed in Microsoft Excel. The raw set of pressure-time histories for all individual elements is not much informative, because in such a form, the solution has too much noise to be valuable in determining the leakage pressure. However, after processing in Excel using the formula ABS(AVERAGEIF(range," $<>0$ ")) to obtain a column of positive average pressure values, the data in Fig. 11 looks much more convenient for understanding with a line representing the fully averaged pressure in the inlet fluid volume throughout the analysis. The averaged water pressure $(\mathrm{kPa})$ vs displacement of plunger (mm) in Fig. 11 also displays the real time of simulation (s) on the secondary axis to show the smooth character of displacement that gradually accelerates and stops to minimise possible dynamic effects. It should be noted that the pressure illustrated in Fig. 11 has been monitored only in the inlet cavity.

The observed volumetric (Fig. 10b) and history fluctuations (Fig. 11) of the liquid can be in the first instance associated with the dynamic type of simulation, then with some compressibility of liquid, and a non-smooth character of the slip of the seal when approaching the critical pressure and associated seal vibrations and waves in the adjacent liquid. A typical stick-slip behaviour (spontaneous jerking motion that can occur while two objects are sliding over each other) can be observed between the top surface of the seal and the top wall in the simulation results animation [22]. The stick-slip becomes very much visually distinctive on approach to the leakage pressure when only the seal edge remains in contact with the wall.

The average pressure history in Fig. 11 is still quite noisy, so the moving average smoothing function with 13 periods is applied to the available time series producing a more clear history of the pressure with less pulsation as illustrated with a solid line in Fig. 11. Analysing the diagram in Fig. 11 and correlating it to the animation [22], two distinctive critical pressure values can be identified. The first one is $\sim 2 \mathrm{kPa}$ corresponding to the plunger displacement of $\sim 5.85 \mathrm{mmm}$ at $\sim 2.9$ s of simulation as highlighted by red lines in Fig. 11. This value ( $\sim 2 \mathrm{kPa})$ is associated with the initiation of leakage and subsequent leak pulses or unsteady leakage. In should be noted that this pressure is not enough to keep the gap between the seal and the wall open. Before reaching this value the fluid pressure increases linearly over time. The stable or steady-state phase of leakage is observed only when reaching a peak pressure in the range of 3-4 kPa starting from $10 \mathrm{~mm}$ of plunger displacement or $\sim 0.4 \mathrm{~s}$ of simulation. This value of pressure $(\sim 4 \mathrm{kPa})$ represents a second critical value that is strong enough to keep the gap open between seal and the wall, thus providing a stable flow of fluid [22].

With the displacement of plunger, the water moves in the same direction creating a pressure on the seal. In its turn, seal reacts to this loading by induction of internal stress and corresponding deformation (see Fig. 10a). The contact between the seal and top wall is frictional with coefficient of friction of 0.3 , that doesn't let the top seal surface to slip easily under increasing water pressure [22]. The hyperelastic nature of seal and its relatively low resistance to the deformation limits the maximum pressure that is achieved in simulation. Therefore, a kind of plateau is observed in Fig. 11 starting from $\sim 9 \mathrm{~mm}$ of plunger displacement or time of $0.385 \mathrm{~s}$. It is associated with a full opening of the gap between the seal and the wall and start of steadystate flow through it. The maximum principal strain in the seal is $\sim 13.5 \%$ when the fluid flow is steady-state as shown in Fig. 9b.

The main purpose of CEL simulation is to obtain the first critical pressure value that indicates the initiation of leakage with pulse leaks. Once we've got it after a double averaging procedure (over the volume and time), the question arises about how reliable this value is. Validation is essential for FE techniques (in- 


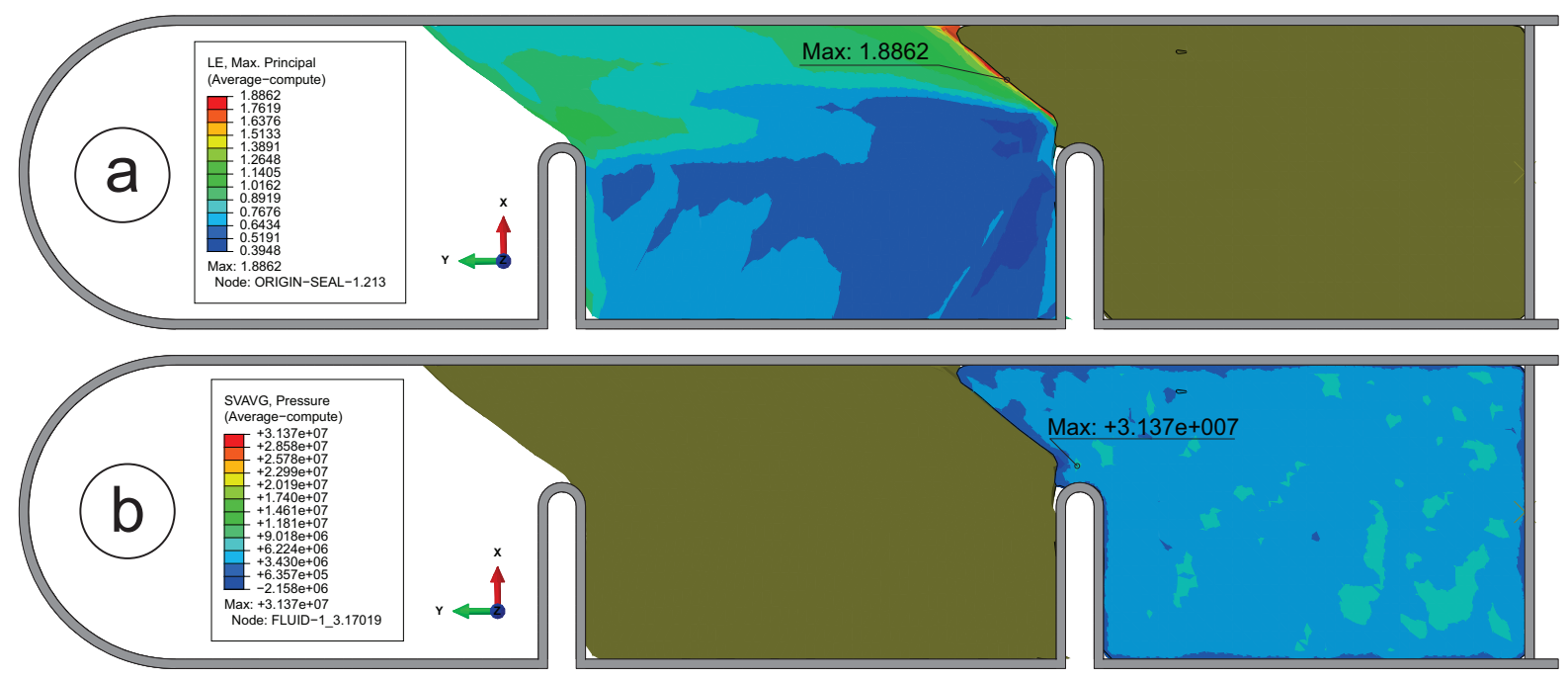

FIG. 13 CURRENTLY BEST AVAILABLE PARTIAL SOLUTION FOR THE FAILURE OF ORIGINAL PACKER BENCHMARK WITH (A) TRUE STRAIN AND (B) FLUID PRESSURE

cluding such advanced as CEL) as it relates the simulated model to the real world and must be carried out to develop confidence in simulation results. One way of carrying out a basic validation is to compare results with, alternative, well validated FE techniques. As experimental validation is difficult and expensive, a validation via a comparative study is chosen for this paper.

The CEL leakage pressure can be validated against a wellestablished leakage prediction technique - Pressure Penetration Loading interaction (PPL) [7]. The PPL simulation was carried out using a similar problem setup to give the same initial configurations and contact conditions. FPP loading was applied to the inlet side surface of the seal using ramping option causing its gradual deformation until start of leakage. One advantage of this technique is the reduced computational time when compared with CEL, static PPL simulations with Abaqus/Implicit are about of order of magnitude faster. The comparison of blow-off pressure obtained with CEL techniques $(2.15 \mathrm{kPa})$ with the one obtained by PPL techniques ( $2.05 \mathrm{kPa})$ gives just a $5 \%$ difference, which can be considered as a good proof of simulation results accuracy. After all it is also possible to validate CEL technique experimentally by simulating the available experimental studies on practical leakage pressure identification such as the one by Liu et al. [23] that will be in focus for future work.

Similar approach is applied to the packers benchmark problems, which are extended from axial symmetry to full 3D considering just $1^{\circ}$ of rotation as shown in Fig. 12 with two elements per thickness of the model and $1 \mathrm{~mm}$ of characteristic element dimension. The sector of $1^{\circ}$ should be theoretically enough to capture the leakage behaviour and minimise the computational effort. Packer, initial fluid volume, moving plunger and stationary walls are all encapsulated into the global Eulerian computational fluid domain, which has to go beyond the Lagrangian components. The type of FE used for the most recent iteration of packer CEL analysis is C3D4 (4-node linear tetrahedron), because C3D8R type used for CEL benchmark above was not robust enough. In this analysis C3D8R was not sufficient for the converged simulations, because of high compressive load induced by fluid. The best currently available partial solution for the failure of original packer benchmark is illustrated in Fig. 13a showing true strain in the packer and Fig. 13b showing fluid pressure. The simulation aborted during the progressive penetration of the fluid into the contact opening between the packer and the borehole. The analysis failure was caused by the excessive distortion of the elements when contacting with fluid - the distortion control with length ration of 0.5 didn't work as it was expected, since tetrahedrons are not susceptible to hourglassing. The elements completely buckled when reaching the peak pressure of about $31 \mathrm{MPa}$ (see Fig. 13b) resulting in unrealistic strains and termination of FEA.

\section{CONCLUSIONS}

The future of leakage prediction may lie in use of the CEL technique as it has many benefits over the alternatives. Not only does this technique allow prediction of critical leakage pressure or blow-off pressure, it also allows for observation of the behaviour of seals after leakage occurs, e.g. steady-state leakage pressure, which will allow for more efficient optimisation of seal design. CEL offers valuable visualisation of leakage mechanisms which enables designers to identify target areas for improvement.

Further work will focus on search of a robust FE-model setup, which would guarantee a stable convergence at high fluid pressure over $1 \mathrm{MPa}$. Explicit simulations with CEL approach [19] are very computationally expensive, e.g. the obtaining the results shown in Fig. 13 required around 2000 CPU-hours, and it is still incomplete. Therefore, further work will also focus on improving the computational efficiency of simulations. Additionally, experimental validation [23] of CEL technique would be extremely valuable when considering the accuracy of the simulated results before application to more costly and complex ap- 
plications. Future work will aim to improve post processing and investigate techniques to reduce noise in the solution such as slightly compressible material models for the operating fluid.

Static implicit analysis if fast, but not robust, on other hand dynamic explicit analysis is robust, but slow. Therefore, taking the best from both solvers, in the form of dynamic implicit analysis, may provide a needed balanced result. Moreover, a specific acoustic type of analysis as a form of FSI is available in dynamic solvers and can be applied to large-deformation enclosures (seals, etc.) with adaptive acoustic meshes for fluids [24].

\section{ACKNOWLEDGMENT}

The authors appreciate the financial support of Weir Group PLC within the WARC project "Design Optimisation of Swell Packers" and University of Strathclyde for hosting during the course of this work. Results were obtained using the EPSRC funded ARCHIE-WeSt High Performance Computer (www.archie-west.ac.uk) EPSRC grant no. $\mathrm{EP} / \mathrm{K} 000586 / 1$.

\section{REFERENCES}

[1] Lou, Y., and Chester, S., 2014. "Kinetics of swellable packers under downhole conditions". Int. J. Appl. Mechanics, 06(06), p. 1450073 [18p].

[2] Gorash, Y., Bickley, A., and Gozalo, F., 2016. "Design optimisation of swellable elastomeric seals using advanced material modelling and FEM simulations". In Poster Int. Conf. on Innovations in Rubber Design (7-8 Dec 2016, London), London: IOM3.

[3] Gorash, Y., Bickley, A., and Gozalo, F., 2017. "Improvement of leak tightness for swellable elastomeric seals through the shape optimization". In Proc. 10th Euro. Conf. on Constitutive Models for Rubbers X - ECCMR X (28-31 Aug 2017, Munich), London: CRC Press, pp. 453-458.

[4] Marks, L., 2013. "Simulation workshop \#1: Multiphysics". Develop3D, Apr 2013, pp. 53-54.

[5] Lorphelin, N., 2015. How to model hygroscopic swelling - COMSOL Blog. www.comsol.com/blogs/how-to-model-hygroscopic-swelling/.

[6] Flory, P. J., and Rehner Jr., J., 1943. "Statistical mechanics of cross-linked polymer networks ii. swelling". The Journal of Chemical Physics, 11(11), pp. 521-526.

[7] Simulia [PPL], 2016. ABAQUS Analysis User's Guide 37.1.7 Pressure penetration loading, Version 2016 ed. Dassault Systèmes Simulia Corp., Providence, RI, USA.

[8] Simulia [AT], 2016. ABAQUS Analysis User's Guide 12.1.1 Adaptivity techniques, Version 2016 ed. Dassault Systèmes Simulia Corp., Providence, RI, USA.

[9] Puri, G., 2011. Python Scripts for Abaqus: Learn by Example. Kan sasana Printer, USA.

[10] Brieger, S., 2016. "Non-parametric optimization". In Bionic Optimization in Structural Design: Stochastically Based Methods to Improve the Performance of Parts and
Assemblies, R. Steinbuch and S. Gekeler, eds. Springer, Berlin, Heidelberg, section 2.6, pp. 37-42.

[11] Wagner, N., and Helfrich, R., 2016. "Topology and shape optimization of structures under contact conditions". In Proc. 1st Euro. Conf: Simulation-Based Optimisation (1213 Oct 2016, Manchester), Hamilton: NAFEMS, pp. 127130.

[12] Simulia [MCP], 2016. Tosca Structure 2016 Documentation - Minimizing contact pressure, Version 2016 ed. Dassault Systèmes Simulia Corp., Karlsruhe, Germany.

[13] Wriggers, P., 2006. "Discretization, large deformation contact”. In Computational Contact Mechanics. Springer, Berlin, Heidelberg, pp. 225-307.

[14] Connolly, S., Gorash, Y., and Bickley, A., 2016. "A comparative study of simulated and experimental results for an extruding elastomeric component". In 23rd Int. Conf. on Fluid Sealing 2016 (2-3 Mar 2016, Manchester), Manchester: BHR Group, pp. 31-41.

[15] Raous, M., 1999. "Quasistatic signorini problem with Coulomb friction and coupling to adhesion". In New Developments in Contact Problems, P. Wriggers and P. Panatiotopoulos, eds., no. 388 in CISM International Centre for Mechanical Sciences. Springer-Verlag, Vienna, chapter 3, pp. 101-178.

[16] Simulia [Comp], 2016. Getting Started with ABAQUS - 10.6.2 Compressibility, Version 2016 ed. Dassault Systèmes Simulia Corp., Providence, RI, USA.

[17] Hossain, M., and Steinmann, P., 2013. "More hyperelastic models for rubber-like materials: consistent tangent operators and comparative study". Journal of the Mechanical Behavior of Materials, 22(1-2), pp. 27-50.

[18] Treloar, L.R.G., 2005. The Physics of Rubber Elasticity, 3rd ed. Oxford University Press, Oxford, UK.

[19] Simulia [CEL], 2017. SIMULIA User Assistance $>$ Abaqus $>$ Abaqus/CAE $>$ Modeling techniques $>$ Eulerian analyses, Version 2017 ed. Dassault Systèmes Simulia Corp., Providence, RI, USA.

[20] Mavrodontis, N., 2017. Modelling hyperelastic behavior using test data in abaqus - Simuleon FEA Blog. https://info.simuleon.com/blog/modelling-hyperelasticbehavior-using-test-data-in-abaqus.

[21] Simulia [EoS], 2017. SIMULIA User Assistance $>$ Abaqus $>$ Materials $>$ Hydrodynamic Properties $>$ Equation of state $>$ Mie-Grüneisen equations of state, Version 2017 ed. Dassault Systèmes Simulia Corp., Providence, RI, USA.

[22] Gorash, Y., 2018. Youtube video-CEL leakage benchmark problem:. www.youtube.com/watch?v=uZB_gYPKHE8.

[23] Liu, Q., Wang, Z., Lou, Y., and Suo, Z., 2014. "Elastic leak of a seal". Extreme Mechanics Letters, 1, pp. 54-61.

[24] van Schalkwijk, R., 1997. "Simulation of noise penetration through car weather seals". In Proc. of ABAQUS Users' Conference (4-6 June 1997, Milan, Italy), Providence, RI: Simulia Corp. 